\title{
Can electrons attract each other without the help of phonons?
}

\section{Electron attraction mediated by Coulomb repulsion}

Authors: A. Hamo, A. Benyamini, I. Shapir, I. Khivrich, J. Waissman, K.

Kaasbjerg, Y. Oreg, F. von Oppen S. Ilani

Nature, 535, 395 (2016)

2. Charge Transfer Excitations, Pair Density Waves, and Superconductivity in Moire Materials

Authors: Kevin Slagle and Liang Fu

arXiv:2003:13690

\section{Correlated double-electron additions at the edge of a two-dimensional electronic system}

Authors: Ahmet Demir, Neal Staley, Samuel Aronson, Spencer Tomarken, Ken West, Kirk Baldwin, Loren Pfeiffer, and Raymond Ashoori

arXiv:2001:09913

\section{Recommended with a Commentary by Patrick A. Lee, MIT}

It is considered well known since the work of Frohlich in the 1950's that electrons can have an effective attraction via the exchange of phonons. Here I collect together a number of papers that show that attraction between electrons is possible using Coulomb interaction between electrons alone. Historically the Frohlich interaction played a key role in the BCS theory of superconductivity, but in hindsight that was the result of an inspired sleight of hand by BCS. Recall that the electron-phonon interaction $g$ has its origin in Coulomb interaction between electrons and ions and is $\sim e^{2}$, and the Frohlich interaction is second order in $g$. In BCS theory, the first order in $e^{2}$ direct Coulomb repulsion was simply ignored. Adding back the Coulomb repulsion will give an interaction that is repulsive at all Matsubara frequencies, (which are the ones that count in BCS or Eliashberg theory, not real frequencies). Only the works by Bogoliubov and Anderson/Morel a few years later justified this step by appealing to the vast difference in energy scale between the phonon and the Fermi energy, leading to a downward renormalization of the Coulomb repulsion relative to the Frohlich attraction.

W. Little wrote an influential paper in 1964 suggesting that a nearby polarizable electronic system can play the role of phonons and give rise to possible high temperature superconductivity. Over half a century later, there is scant evidence that this mechanism works in practice. Part of the difficulty is that electronic systems typically do not enjoy the separation of energy scale that was crucial for the phonon induced pairing mechanism. In the 
mean time, it is now commonly accepted that non s-wave pairing is possible with Coulombic repulsion. Nevertheless, it is interesting to go back and ask a more stringent question that does not rely on dynamical polarizibility: can two electrons attract even in the static $(\omega=0)$ limit?

The paper by Homo et al. answered this question in the positive by designing a minimal set-up based on carbon nanotubes. They create a double well quantum dot in the first nanotube using gates and place another double well quantum dot nearby to function as Little's polarizer. Because they have exquisite control of the potentials seen by the electrons, they demonstrated that while normally electrons will occupy the quantum dot one by one, there is a gate parameter regime where the first quantum dot is either empty or accommodate a pair of electrons, i.e. it functions as a negative $U$ center. They emphasize that what is important is the discreteness of charge, i.e. the charge density on an electron does not repel itself.

It is challenging to see how this bottom up strategy can be extended to create arrays of negative U centers. A recent paper by Slagle and Fu proposes how to take this next step, at least theoretically. They take as inspiration the physical system of moire unit cell formed by stacking monolayers of $W S_{2}$ and $W S e_{2}$, but the mathematical model is a simple one, based on a honeycomb lattice where the $\mathrm{A}$ and $\mathrm{B}$ sites in the unit cell have different potentials. Let us fill the system with holes and assume that site A (colored black in Fig. 1) is more favorable. With large on-site repulsion, the system forms a Mott insulator (more properly it should be called a charge transfer insulator) when there is one hole per unit cell. The holes will reside in site A, forming a triangular lattice. Now introduce extra holes (we refer to them as doped holes) and ask where they will go. Shown in Fig. 1 are 3 options. The simplest one is that the hole occupies the B site (colored red). A better option may be that this hole pushes the neighboring hole out of the way, forming a dipole and enjoys the polarization energy. This is like the electronic polarizer envisioned by Little. But why stop there? Slagle and Fu pointed to a third option, where three B sites are occupied, together with a central vacancy on the A site. Note that there is a net addition of 2 electrons in this configuration. They call this a trimer and showed that with realistic Coulomb interaction, there is a regime where the trimer cost less energy than two spatially separated single hole that are in either configuration 1 or 2 . Thus the trimer serves as a negative $U$ center. Note that this just follows from electrostatics and does not invoke fancy retardation effects.

Slagle and $\mathrm{Fu}$ went on to show that with long range Coulomb repulsion, a finite density of these trimers will form a Wigner crystal of pairs. These structures will have commensurate periodicities which are different from what one expects from a Wigner crystal of single doped holes. This is something that can be tested exprimentally in the $W S_{2} / W S e_{2}$ system.

Of course, Slagle and Fu could not resist asking whether superconductivity will arise with a finite but low concentration of holes. To do this the trimer has to be mobile. The hopping of the trimer itself is suppressed because several intermediates steps with large energy denominators are involved. A more favorable situation occurs at a doping level where a Fermi sea of single holes co-exists with trimers and hybridize with them. In this case a hopping term where a trimer boson turns into a pair of Fermionic holes is introduced. Slagle and Fu made an analogy with the Feshbach resonance in atomic physics. What is clear is that this model contains a cross-over between the BCS and BEC limit, the latter obtains when the trimer Bose condense. The pair hopping term leads to a natural mean field 


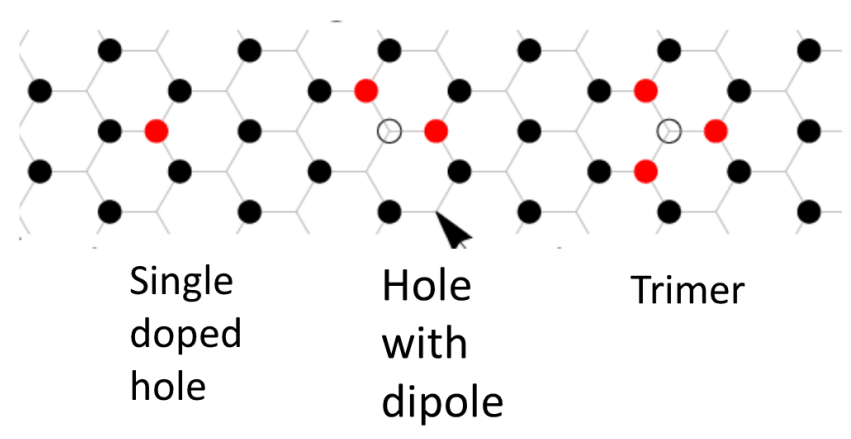

Figure 1: From Slagle and Fu, The honeycomb lattice is halved filled by holes which fully occupy the A sites (colored black). Additional doped holes may have one of three configurations illstrated here: a single hole on the $\mathrm{B}$ site (colored red), a hole with a neighboring dipole, or a trimer configuration where 3 red holes surround a vacancy in site A. Note that a trimer adds a pair of holes.

decoupling which is described in the appendix, showing that unlike BCS, a large energy gap which is not exponentially dependent on the coupling constant can occur.

It should be noted that in the BEC limit, the energy gap may be large, but $T_{c}$ is controlled by the kinetic energy of the Bosons, which can be much smaller. Slagle and Fu did not discuss $T_{c}$ in their paper. It turns out that the same model with negative $\mathrm{U}$ boson hybridized with a fermion band has been worked out in the old literature in different contexts. The negative $\mathrm{U}$ centers were treated either as randomly localized states [1] or periodic [2]. Their conclusion is that the $T_{c}$ to energy gap ratio is not universal as in BCS in these mean field theories and is generally much smaller. So maybe this may not be a profitable route to high $T_{c}$ after all. There remains room for further exploration.

As long as we are pursuing physics archaeology, there is a long history of appealing to negative U centers as a path to pairing. C. M. Varma [3] in 1988 put together a set of "valence skipping" ions as examples of negative U centers. PbTe is a narrow gap semiconductor, but become superconducting with $T_{c}$ up to $1.4 \mathrm{~K}$ when doped with a small concentration of $\mathrm{Tl}$, which is one of the valence skipping ions.[4] Dzero and Schmalian [6] provided an interesting theoretical description using the same boson/Fermion-pair hybridization model. Unfortunately the experimentalists decided that the superconductivity appears to be conventional upon further scrutiny and the subject fizzled out.[5]

Returning to the topic of adding charges to a quantum dot, I would like to recommend a third paper which reports pairing phenomena in the edge state of a large quantum dot subject to a magnetic field. Demir et al used the technique of single electron capacitance spectroscopy to study the charge addition spectrum of a quantum dot. A large 2D quantum $\operatorname{dot}$ ( $800 \mathrm{~nm}$ diameter) is placed between two electrodes of a capacitor, and tunnel coupled to one of them, which serves as a reservoir. When the gate voltage across the capacitor is varied, a single electron tunnel from the reservoir to the dot. Its image charge produces a spike in the 

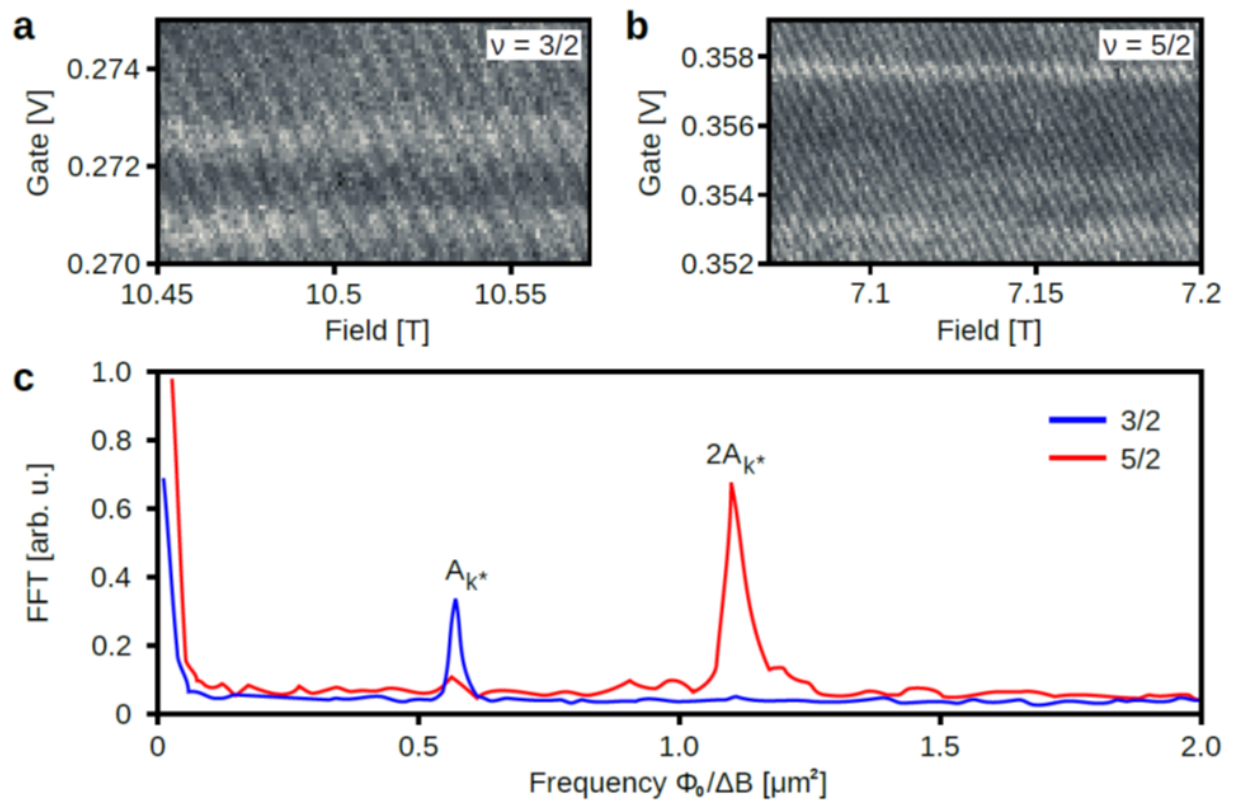

Figure 2: From Demir et al. Lines in (a) shows the energy to add an electron one at a time to the edge of a quantum dot as a function of magnetic field, when the Landau level filling is $3 / 2$. (b) At filling $5 / 2$ the magnetic field between lines for a fixed gate voltage has been halved. (c) Fourier transform of the B dependence at a fixed gate voltage. Not only is the frequency doubled, the amplitude has also doubled, a direct indication that electrons are enter the quantum dot in pairs,

capacitance. By measuring the capacitance as a function of gate voltage and magnetic field, the addition of thousands of electrons to the dot can be monitored, one electron at a time, a remarkable feat in itself. In Fig 2, the vertical axis is the gate voltage and the horizontal axis is the magnetic field. Each line marks the ground state energy of a system with a fixed number of electrons. The fact that all the lines decreases with increasing magnetic field is taken as evidence that the electrons are entering the dot by occupying the edge states. This is expected even when the system is not at integer filling and is compressible, because the tunneling rate into the interior of the dot is known to be suppressed by Coulomb blockade effect. For filling less than $\nu=2$, i.e. when the $\mathrm{N}=0$ Landau level is being filled, the lines are evenly spaced and the periodicity in $\mathrm{B}$ is given by the condition that flux through the dot is increasing in units of the flux quantum $h c / e$. An example is shown for $\nu=3 / 2$ on the left of Fig 2. The surprising discovery made by Demir et al. is that at filling $\nu=5 / 2$, the period in $\mathrm{B}$ field has been reduced by half. So the condition for charge addition is for the flux to increase by $h c / 2 e$. Furthermore, the amount of charge added can be measured by the size of the spikes, which has gone up by a factor of two. Both these features are evident in the Fourier transform of the signal: both the frequency and the height has doubled. It is apparent that electrons are entering the dots in pairs. Demir et al report observing this phenomenon whenever the filling factor is between 2 and 5 . Away from 5/2, there is a further 
effect that the lines are bunched in pairs, that is the pairs themselves have a double period.

It should be noted that the way pairs enter is not described by a naive weak coupling negative U picture, by which I mean a situation where the single electron structure of the edge state remains, but electrons are occupying these states 2 at a time. This will give double height spikes but with a period in B that is doubled, in contrast to the observed halving of the period. Apparently compared with the $\nu=3 / 2$ case, 4 electrons enter the dot when the flux has increased by one flux quantum $h c / e$, as opposed to one. A simple explanation is that electrons have formed charge $2 \mathrm{e}$ bound states. The question is where does this strong binding come from?

The formation of charge $2 \mathrm{e}$ bound states at the edge of a quantum Hall state have been reported earlier by the group of Morty Heiblum. [7, 8] (See commentary by Felix von Oppen, November 2014) They utilized an interference device to isolate the outermost edge state. They also reported shot noise in units of 2e. Compared with this earlier pioneering work, Demir's et al provide information on the ground state energy as a function of charge number and the setup is more straight forward to interpret. For the theorist this has become a challenging question that is difficult to evade.

For completeness I mention the observation of multiple electrons (why stop at two?) entering a narrow channel at the interface of $\mathrm{LaAlO}_{3}$ and $\mathrm{SrTiO}_{3}$ by the group of Jeremy

Levy. See [9] and earlier references. This is a system near a ferro-electric transition and is a different story, better saved for a different time.

\section{References}

[1] A. G. Malshukov, Solid state comm. 77, 57, (1991).

[2] Y. Bar-Yam, Phys. Rev. B43, 359 (1991).

[3] C. M. Varma Phys. Rev. Lett. 61, 2713 (1988).

[4] Y. Matsushita, H. Bluhm, T. H. Geballe and I. R. Fisher Phys. Rev. Lett. 94,157002 (2005).

[5] Y. Matsushita et al. Phys. Rev. B 74, 134512 (2006).

[6] M. Dzero and J. Schmalian Phys. Rev. Lett. 94.157003 (2005).

[7] H. K. Choi et al, Nature Comm. 6, 7435 (2015).

[8] I. Sivan et al, Phys. Rev. B 97, 125405 (2018).

[9] M. Briggeman et al., Science 367, 769 (2020). 